\title{
"LazImpa": Lazy and Impatient neural agents learn to communicate efficiently
}

\author{
Mathieu Rita $^{1} \quad$ Rahma Chaabouni $^{1,2} \quad$ Emmanuel Dupoux $^{1,2}$ \\ ${ }^{1}$ Cognitive Machine Learning (ENS/EHESS/PSL Research University/CNRS/INRIA) \\ ${ }^{2}$ Facebook AI Research \\ mathieu.rita@polytechnique.edu, \{rchaabouni,dpx\}@fb.com
}

\begin{abstract}
Previous work has shown that artificial neural agents naturally develop surprisingly nonefficient codes. This is illustrated by the fact that in a referential game involving a speaker and a listener neural networks optimizing accurate transmission over a discrete channel, the emergent messages fail to achieve an optimal length. Furthermore, frequent messages tend to be longer than infrequent ones, a pattern contrary to the Zipf Law of Abbreviation (ZLA) observed in all natural languages. Here, we show that near-optimal and ZLA-compatible messages can emerge, but only if both the speaker and the listener are modified. We hence introduce a new communication system, "LazImpa", where the speaker is made increasingly lazy, i.e., avoids long messages, and the listener impatient, i.e., seeks to guess the intended content as soon as possible.
\end{abstract}

\section{Introduction}

Recent emergent-communication studies, renewed by the astonishing success of neural networks, are often motivated by a desire to develop neural network agents eventually able to verbally interact with humans (Havrylov and Titov, 2017; Lazaridou et al., 2017). To facilitate such interaction, neural networks' emergent language should possess many natural-language-like properties. However, it has been shown that, even if these emergent languages lead to successful communication, they often do not bear core properties of natural language (Kottur et al., 2017; Bouchacourt and Baroni, 2018; Lazaridou et al., 2018; Chaabouni et al., 2020).

In this work, we focus on one basic property of natural language that resides on the tendency to use messages that are close to the informational optimum. This is illustrated in the Zipf's law of Abbreviation (ZLA), an empirical law that states that in natural language, the more frequent a word is, the shorter it tends to be (Zipf, 1949; Teahan et al., 2000; Sigurd et al., 2004; Strauss et al., 2007). Crucially, ZLA is considered to be an efficient property of our language (Gibson et al., 2019). Besides the obvious fact that an efficient code would be easier to process for us, it is also argued to be a core property of natural language, likely to be correlated with other fundamental aspects of human communication, such as regularity and compositionality (Kirby, 2001). Encouraging it might hence lead to emergent languages that are also more likely to develop these other desirable properties.

Despite the importance of such property, Chaabouni et al. (2019) showed that standard neural network agents, when trained to play a simple signaling game (Lewis, 1969), develop an inefficient code, which even displays an anti-ZLA pattern. That is, counterintuitively, more frequent inputs are coded with longer messages than less frequent ones. This inefficiency was related to neural networks' "innate preference" for long messages. In this work, we aim at understanding which constraints need to be introduced on neural network agents in order to overcome their innate preferences and communicate efficiently, showing a proper ZLA pattern.

To this end, we use a reconstruction game where we have two neural network agents: speaker and listener. For each input, the speaker outputs a sequence of symbols (which constitutes the message) sent to the listener. The latter needs then to predict the speaker's input based on the given message. Also, similarly to the previous work, inputs are drawn from a power-law distribution.

We first describe the experimental and optimization framework (see Section 2). In particular, we introduce a new communication system called 'LazImpa', comprising two different constraints (a) Laziness on the speaker side and (b) Impatience on the listener side. The former constraint is inspired by the least-effort principle which is attested to be a ubiquitous pressure in human communication (Piantadosi et al., 2011; Zipf, 1949; Kanwal et al., 2017).

However, if such a constraint is applied too early, the system does not learn an efficient system. We show that incrementally penalizing long messages in the cost function enables an early exploration of the message space (a kind of 'babbling phase') and prevents converging to an inefficient local minimum.

The other constraint, on the listener side, relies on the prediction mechanism, argued to be important in language comprehension (e.g., Federmeier, 2007; Altmann and Mirković, 2009), and is achieved by allowing 
the listener to reconstruct the intended input as soon as possible. We also provide a two-level analytical method: first, metrics quantifying the efficiency of a code; second, a new protocol to measure its informativeness (see Section 3). Applying these metrics, we demonstrate that, contrary to the standard speaker/listener agents, our new communication system 'LazImpa' leads to the emergence of an efficient code. The latter follows a ZLA-like distribution, close to natural languages (see Sections 4.1 and 4.2). Besides the plausibility of the introduced constraints, our new communication system is, first, taskand architecture-agnostic (requires only communicating with sequences of symbols), and second allows stable optimization of the speaker/listener. We also show how both listener and speaker constraints are fundamental to the emergence of a ZLA-like distribution, as efficient as natural language (see Section 4.3).

\section{Experimental framework}

We explore the properties of emergent communication in the context of referential games where neural network agents, Speaker and Listener, have to cooperatively communicate in order to win the game.

Speaker network receives an input $i \in \mathcal{I}$ and generates a message $m$ of maximum length max_len. The symbols of the message belong to a vocabulary $V=$ $\left\{s_{1}, s_{2}, \ldots, s_{\text {VOC_size-1 }}\right.$, EOS $\}$ of size Voc_size where EOS is the 'end of sentence' token indicating the end of Speaker's message. Listener network receives and consumes the message $m$. Based on this message, it outputs $\hat{i}$. The two agents are successful if Listener manages to guess the right input (i.e., $\hat{i}=i$ ).

We make two main assumptions. First inputs are drawn from $\mathcal{I}$ following a power-law distribution, where $\mathcal{I}$ is composed of 1000 one-hot vectors.

Consequently, the probability of sampling the $k^{t h}$ most frequent input is: $\frac{1 / k}{\sum_{j=1}^{1000} 1 / j}$ modelling words' distribution in natural language (Zipf, 2013) (see details in Appendix A.1.1). Second, we experiment in the main paper with max_len $=30$ and voc_size $=40 .{ }^{1}$ We further discuss the influence of these assumptions in Appendix. A.4.2 and show the robustness of our results to assumptions change.

In our analysis, we only consider the successful runs, i.e., the runs with a uniform accuracy strictly higher than 97\% over all possible 1000 inputs. An emergent language consists then of the input-message mapping. That is, for each input $i \in \mathcal{I}$ fed to Speaker after successful communication, we note its output $m$.

By $\mathcal{M}$, we define the set of messages $m$ used by our agents after succeeding in the game.

\footnotetext{
${ }^{1}$ This combination makes our setting comparable to natural languages; the latter has no upper bound on the maximum length, also a vocabulary size of 40 is close to the alphabet size of the natural languages we study of mean vocabulary size equal to 41.75. See Chaabouni et al. (2019) for more details.
}

\subsection{Agent architectures}

In our experiments, we compare two communication systems:

- Standard Agents: as a baseline, composed of Standard Speaker and Standard Listener;

- 'LazImpa': composed of Lazy Speaker and Impatient Listener.

For both Speaker and Listener, we experiment with either standard or modified LSTM architectures (Hochreiter and Schmidhuber, 1997).

\subsubsection{Standard Agents}

Standard Speaker. Standard Speaker is a singlelayer LSTM. First, Speaker's inputs $i$ are mapped by a linear layer into an initial hidden state of Speaker's LSTM cell. Then, the message $m$ is generated symbol by symbol: the current sequence is fed to the LSTM cell that outputs a new hidden state. Next, this hidden state is mapped by a linear layer followed by a softmax to a Categorical distribution over the vocabulary. During the training phase, the next symbol is sampled from this distribution. During the testing phase, the next symbol is deterministically selected by taking the argmax of the distribution.

Standard Listener. Standard Listener is also a singlelayer LSTM. Once the message $m$ is generated by Speaker, it is entirely passed to Standard Listener. Standard Listener consumes the symbols one by one, until the EOS token is seen (the latter is included and fed to Listener). At the end, the final hidden state is mapped to a Categorical distribution $L(m)$ over the input indices (linear layer + softmax). This distribution is then used during the training to compute the loss. During the testing phase, we take the argmax of the distribution as a reconstruction candidate.

Standard loss $\mathcal{L}_{s t d}$. For Standard Agents, we merely use the cross-entropy loss between the ground truth onehot vector $i$ and the output Categorical distribution of Listener $L(m)$.

\subsubsection{LazImpa}

Lazy Speaker. Lazy Speaker has the same architecture as Standard Speaker. The 'Laziness' comes from a cost on the length of the message $m$ directly applied to the loss.

Impatient Listener. We introduce Impatient Listener, designed to guess the intended content as soon as possible. As shown in Figure 1, Impatient Listener consists of a modified Standard Listener that, instead of guessing $i$ after consuming the entire message $m=\left(m_{0}, \ldots, m_{t}\right)$, makes a prediction $\hat{i}_{k}$ for each symbol $m_{k}{ }^{2}$ This modification takes advantage of the recurrent property of the LSTM, however, could be adapted to any causal sequential neural network model.

\footnotetext{
${ }^{2} m_{t}=\mathrm{EOS}$ by construction.
} 
At training, a prediction of Impatient Listener, at a position $k$, is a Categorical distribution $L\left(m_{: k}\right)$, constructed using a shared single linear layer followed by a softmax (with $m_{: k}=\left(m_{0}, \ldots, m_{k}\right)$ ). Eventually, we get a sequence of $t+1$ distributions $L(m)=$ $\left(L\left(m_{: 0}\right), \ldots, L\left(m_{: t}\right)\right)$, one for each reading position of the message.

At test time, we only take the argmax of the distribution generated by Listener when it reads the EOS token.

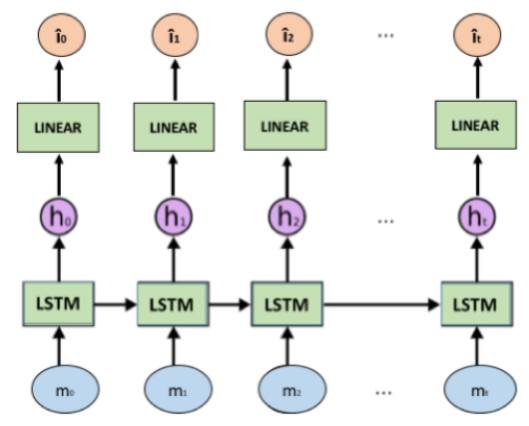

Figure 1: Impatient Listener architecture. The agent is composed of a single-layer LSTM cell and one shared linear layer followed by a softmax. It generates a prediction at each time step.

LazImpa Loss $\mathcal{L}_{l a z}$. LazImpa loss is composed of two parts that model 'Impatience' $\left(\mathcal{L}_{l a z / L}\right)$ and 'Laziness' $\left(\mathcal{L}_{\text {laz } / S}\right)$, such that,

$$
\mathcal{L}_{l a z}(i, m, L(m))=\mathcal{L}_{l a z / L}(i, L(m))+\mathcal{L}_{l a z / S}(m)
$$

On one hand, $\mathcal{L}_{l a z / L}$ forces Impatient Listener to guess the right candidate as soon as possible when reading the message $m$. For this purpose, with $i$ the ground-truth input and $L(m)=\left(L\left(m_{: 0}\right), \ldots, L\left(m_{: t}\right)\right)$ the sequence of intermediate distributions, the Impatience Loss is defined as the mean cross-entropy loss between $i$ and the intermediate distributions:

$$
\mathcal{L}_{l a z / L}(i, L(m))=\frac{1}{t+1} \sum_{k=0}^{t} \mathcal{L}_{s t d}\left(i, L\left(m_{: k}\right)\right),
$$

Hence, all the intermediate distributions contribute to the loss function according to the following principle: the earlier the Listener predicts the correct output, the larger the reward is.

On the other hand, $\mathcal{L}_{l a z / S}$ consists of an adaptive penalty on message lengths. The idea is to first let the system explore long and discriminating messages (exploration step) and then, once it reaches good enough communication performances, we apply a length cost (reduction step). With $|m|$ the length of the message associated with the input $i$ and 'acc' the estimation of the accuracy (proportion of inputs correctly communicated weighted by appearance frequency), the Laziness Loss is defined as:

$$
\mathcal{L}_{\text {laz/S }}(m)=\alpha(\operatorname{acc})|m|
$$

To schedule this two-step training, we model $\alpha$ as shown in Figure 2. The regularization is mainly composed of two branches: (1) exploration step and (2) reduction step. The latter starts only when the two agents become successful.

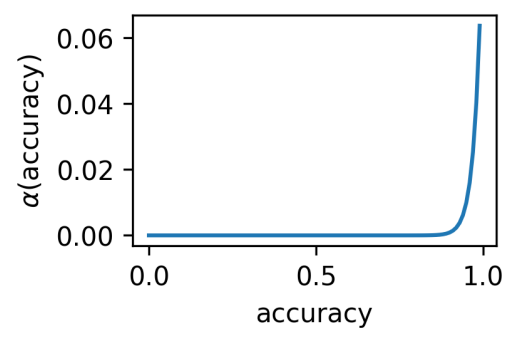

Figure 2: Scheduling of the regularization parameter $\alpha$ as a function of the accuracy. We distinguish two different regimes: the exploration and the reduction regimes. See the mathematical description in Appendix A.1.4

\subsection{Optimization}

The overall setting, which can be seen as a discrete autoencoder, cannot be differentiated directly, as the latent space is discrete. We use a hybrid optimization between REINFORCE for Speaker (Williams, 1992) and classic back-propagation for Listener (Schulman et al., 2015).

With $\mathcal{L}$ the loss of the system, $i$ the ground-truth input and $L(m)$ the output distribution of Listener that takes the message $m$ as input, the training task consists in minimizing the expectation of the loss $\mathbb{E}[\mathcal{L}(i, L(m))]$. The expectation is computed w.r.t the joint distribution of the inputs and the message sequences. Let's denote $\theta_{L}$ and $\theta_{S}$ Listener and Speaker parameters respectively. The optimization task requires to compute the gradient $\nabla_{\theta_{S} \cup \theta_{L}} \mathbb{E}[\mathcal{L}(i, L(m))]$. An unbiased estimate of this gradient is the gradient of the following function:

$$
\mathbb{E}[\underbrace{\mathcal{L}\left(i, L\left(m ; \theta_{L}\right)\right)}_{(A)}+\underbrace{\left(\left\{\mathcal{L}\left(i, L\left(m ; \theta_{L}\right)\right)\right\}-b\right) \log P_{S}\left(m \mid \theta_{S}\right)}_{(B)}]
$$

where $\{$.$\} is the stop-gradient operation, P_{S}\left(m \mid \theta_{S}\right)$ the probability that Speaker generates the message $m$, $b$ the running-mean baseline used to reduce variance (Williams, 1992). We also promote exploration by encouraging Speaker's entropy (Williams and Peng, 1991).

The gradient of (4) w.r.t $\theta_{L}$ is found via conventional back-propagation (A) while gradient w.r.t $\theta_{S}$ is found with a REINFORCE-like procedure estimating the gradient via a Monte-Carlo integration calculated over samples of the messages (B). Once the gradient is estimated, it is eventually passed to the Adam optimizer (Kingma and $\mathrm{Ba}, 2014)$. 
In Appendix A.3.1, we show that LazImpa leads to a stable convergence. We use the EGG toolkit (Kharitonov et al., 2019) as a starting framework. For reproducibility, the code can be found at https:// github.com/MathieuRita/Lazimpa and the set of hyper-parameters used is presented in Appendix A.1.

\section{Analytical method}

As ZLA is defined informally, we first introduce reference distributions for comparison. Then, we propose some simple metrics to evaluate the overall efficiency of our emergent codes. Eventually, we provide a simple protocol to analyze the distribution of information within the messages.

\subsection{Reference distributions}

We compare the emergent languages to the reference distributions introduced in Chaabouni et al. (2019). We provide below a brief description of the different distributions, however, we invite readers to refer to the reference paper for more details.

Optimal Coding (Cover and Thomas, 2006) guarantees the shortest average message length with max_len $=30$ and voc_size $=40$. To do so, we deterministically associate the shortest messages to the most frequent inputs. See Ferrer i Cancho et al. (2013) for more details about the derivation of Optimal Coding.

Natural Language We also compare emergent languages with several human languages. In particular, we consider the same languages of the reference paper (English, Arabic, Russian, and Spanish). These references consist of the mapping from the frequency of the top 1000 most frequent words in each language to their length (approximated by the number of characters of each word). ${ }^{3}$

\subsection{Efficiency metrics}

In this work, we examine the constraints needed for neural agents to develop efficient languages. We use three metrics to evaluate how efficient the different codes are. For all metrics, $N$ denotes the total number of messages $(=1000)$ and $l(m)$ the length of a message $m$.

Mean message length $L_{t y p e}$ : measures the mean length of the messages assuming a uniform weight for each input/message:

$$
L_{\text {type }}=\frac{1}{N} \sum_{m \in \mathcal{M}} l(m),
$$

Mean weighted message length $L_{\text {token }} \quad$ : measures the average length of the messages weighted by their generation frequency:

$$
L_{\text {token }}=\sum_{m \in \mathcal{M}} p(m) l(m)
$$

\footnotetext{
${ }^{3}$ We use the frequency lists from http://corpus. leeds.ac.uk/serge/.
}

where $p(m)$ is the probability of message $m$ (equal to the probability of input $i$ denoted by $m$ ) such that $\sum_{m \in \mathcal{M}} p(m)=1$. Formally, the message $m$ referring to the $k^{t h}$ most frequent input would have a probability $\frac{1 / k}{\sum_{1}^{1000} 1 / j}$.

Note that, the Optimal Coding is the one that minimizes $L_{\text {token }}$ (Cover and Thomas, 2006; Ferrer i Cancho et al., 2013).

ZLA significance score $p_{Z L A}$ : Let's note $\left(l_{i}\right)_{i \in \mathcal{I}}$ a distribution of message lengths of a code. As a ZLA distribution is the one that minimizes $L_{\text {token }}$, we can check if $\left(l_{i}\right)_{i \in \mathcal{I}}$ follows ZLA by testing if its $L_{\text {token }}$ is lower than any random permutation of its frequencylength mapping. This is the idea of the randomization test proposed by Ferrer i Cancho et al. (2013).

The test checks whether $L_{\text {token }}$ coincides with $\sum_{i \in \mathcal{I}} l_{i} f_{\sigma(i)}$, with $\sigma(i)$ a random permutation of inputs. We can eventually compute a p-value $p_{Z L A}$ (at threshold $\alpha$ ) that measures to which extent $L_{\text {token }}$ is likely to be smaller than any other weighted mean message length of a frequency-length mapping. $p_{Z L A}<\alpha$ indicates that any random permutation would have most likely longer weighted mean length. Thus $\left(l_{i}\right)_{i \in \mathcal{I}}$ follows significantly a ZLA distribution. Additional details are provided in Appendix A.3.2.

\subsection{Information analysis}

We also provide an analytical protocol to evaluate how information is distributed within the messages. We consider a symbol to be informative if replacing it randomly has an effect on Listener's prediction. Formally, let's take the message $m=\left(m_{0}, \ldots, m_{t}\right)$ associated to the ground truth input $i$ after training. To evaluate the information contained in the symbol at position $k, m_{k}$, we substitute it randomly by drawing another symbol $r_{k}$ uniformly from the vocabulary (except the EOS token). Then, we feed this new message $\tilde{m}=\left(m_{1}, \ldots, r_{k}, \ldots, m_{t}\right)$ into Listener that outputs $\tilde{o}_{m, k}$ (index $m$ indicates that the original message was $m$, index $k$ indicates that the $k^{t h}$ symbol of the original message has been replaced). We define $\Lambda_{m, k}$ a boolean score that evaluates whether the symbol replaced at position $k$ has an impact on the prediction, such that $\Lambda_{k, m}=\mathbf{1}\left(\tilde{o}_{m, k} \neq i\right)$. If $\Lambda_{m, k}=1$, the $k^{t h}$ symbol of message $m$ is considered as informative. If $\Lambda_{m, k}=0$, it is considered as non-informative. We do not consider misreconstructed inputs, neither the position $t$, as $m_{t}=\mathrm{EOS} .{ }^{4}$ This token is needed for Listener's prediction at test time.

This test allows us to introduce some variables that quantify to which extent information is effectively distributed within the messages. As previously, we note $l(m)$ the length of message $m$ and $N$ the total number of messages.

\footnotetext{
${ }^{4}$ As we only consider successful runs, more than $97 \%$ of inputs are, by definition, well-reconstructed.
} 


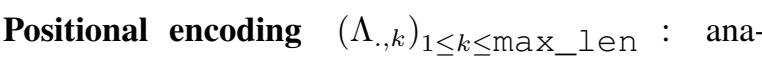
lyzes the position of informative symbols within an emergent code. We assign a score $\Lambda_{., k}$ for each position $k$ that counts the proportion of informative symbols over all the messages of a language:

$$
\Lambda_{., k}=\frac{1}{N(k)} \sum_{m \in \mathcal{M}} \Lambda_{m, k},
$$

where $N(k)$ is the number of messages that have a symbol (different from EOS) at position $k$.

Effective length $L_{e f f}$ : measures the mean number of informative symbols by message:

$$
L_{e f f}=\frac{1}{N} \sum_{m \in \mathcal{M}} \sum_{k=1}^{l(m)-1} \Lambda_{m, k} .
$$

$L_{e f f}$ counts the average number of symbols Listener relies on (removing all the uninformative symbols for which $\left.\Lambda_{m, k}=0\right)$. A message with only informative symbols would have $L_{\text {eff }}=L_{\text {type }}-1 .^{5}$

Information density $\rho_{\text {inf }}:$ measures the fraction of informative symbols in a language:

$$
\rho_{i n f}=\frac{1}{N} \sum_{m \in \mathcal{M}} \frac{1}{l(m)-1} \sum_{k=1}^{l(m)-1} \Lambda_{m, k} .
$$

We integrate over the first $l(m)-1$ positions as we disregard EOS that occurs in all messages. ${ }^{6} 0 \leq \rho_{\text {inf }} \leq$ 1. If $\rho_{\text {inf }}=1$, messages are limited to the informative symbols (all used by Listener to decode the message). The lower $\rho_{\text {inf }}$ is, the more non-informative symbols are in the message.

As we do not have Listener when generating Optimal Coding, we compute these metrics for the latter reference by considering all symbols, but EOS, informative.

\section{Results}

In this section, we study the code of our new communicative system, LazImpa, and compare it to the Standard Agents baseline and the different reference distributions. We show that LazImpa leads to near-optimal and ZLA-compatible languages. Eventually, we demonstrate how both Impatience and Laziness are required to get human-level efficiency. All the quantitative results of the considered codes are gathered in Table 1.

\subsection{LazImpa vs. Standard Agents}

We compare here LazImpa to the baseline system Standard Agents both in terms of the length efficiency and the allocation of information.

\footnotetext{
${ }^{5}$ We subtract 1 as we disregard EOS in all messages.

${ }^{6} \mathrm{By}$ convention, for the case where $m=(\mathrm{EOS}), \frac{0}{0}=1$.
}

Length efficiency of the communication. Contrary to Standard Agents, LazImpa develops an efficient communication as presented in Figure 3. Indeed, its average length of the messages is significantly lower than the Standard Agents system (average $L_{\text {type }}=29.6$ for Standard Agents vs. $L_{t y p e}=5.49$ for LazImpa). The latter demonstrates length distributions almost constant and close to the maximum length we set $(=30)$. We demonstrate in Appendix A.2.1 how the exploration of long messages in Standard Agents is key for agents' success in the reconstruction game, even though, in theory, shorter messages are sufficient.

Interestingly, both systems do not only differ by their average length, but also by the distribution of messages length. Specifically, the Standard Agents system follows significantly an anti-ZLA distribution (see Appendix A.3.2 for quantitative support of this claim) while LazImpa has an average $L_{\text {token }}=3.78$ showing a ZLA pattern: the shortest messages are associated to the most frequent inputs. The randomization test gives quantitative support of this observation $\left(p_{Z L A}<10^{-5}\right)$.

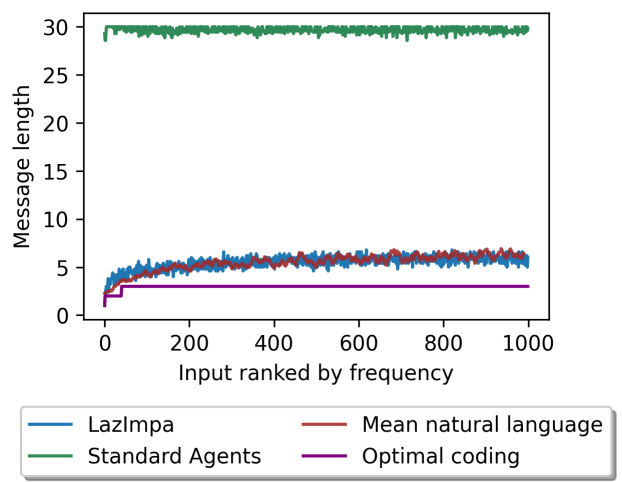

Figure 3: Average message length across successful runs as a function of input frequency rank.

Informativeness of the communication. When considering how Standard Agents system allocates information, shown in Figure 4a, we can make two striking observations. First, only a very small part of the messages are informative (on average $\rho_{\text {inf }}=11 \%$ ). Therefore, even if long messages seem necessary for the agents to succeed, most of the symbols are not used by Listener. In particular, if $L_{\text {type }}=29.6$ on average, the average number of symbols used by Standard Listener $\left(L_{\text {eff }}\right)$ is only equal to 3.33 (which is even smaller than natural languages' mean message length $L_{\text {type }}=5.46$ ). Surprisingly, we also observe that, if we restrict the messages to their informative symbols (i.e. removing positions $k$ with $\Lambda_{k, .}=0$ ), the length statistics follow a ZLA-like distribution (see Figure 9 in Appendix A.2.2). Second, in all our experiments, the information is localized at the very end of the messages. That is, there is almost no actual information in the messages about Speaker's inputs before the last symbols.

Contrarily, Figure 4d shows a completely different 


\begin{tabular}{|c|c||c|c|c||c|c|}
\hline Class & Code & $L_{\text {type }}$ & $L_{\text {token }}$ & $p_{Z L A}$ & $L_{\text {eff }}$ & $\rho_{\text {inf }}$ \\
\hline Emergent & Standard Agents & $29.6 \pm 0.4$ & $29.91 \pm 0.07$ & $>1-10^{-5}$ & $3.33 \pm 0.46$ & $0.11 \pm 0.02$ \\
& LazImpa & $5.49 \pm 0.67$ & $3.78 \pm 0.34$ & $<10^{-5 *}$ & $2.67 \pm 0.07$ & $0.60 \pm 0.07$ \\
\hline \hline References & Mean natural languages & $5.46 \pm 0.61$ & $3.55 \pm 0.14$ & $<10^{-5 *}$ & $/$ & $/$ \\
& Optimal Coding & 2.96 & 2.29 & $<10^{-5 *}$ & 1.96 & 1.00 \\
\hline
\end{tabular}

Table 1: Efficiency and information analysis of emergent codes and reference distribution. For each metric, we report the mean value and the standard deviation when relevant (across seeds when experimenting with emergent languages and across the natural languages presented in Section 3.1 for Mean natural languages). $L_{\text {type }}$ is the mean message length, $L_{\text {token }}$ is the mean weighted message length, $p_{Z L A}$ the ZLA significance score, $L_{e f f}$ the effective length and $\rho_{\text {inf }}$ the information density. ' $/$ ' indicates that the metric cannot be computed. For $p_{Z L A}$, '*' indicates that the $\mathrm{p}$-value is significant $(<0.001)$.

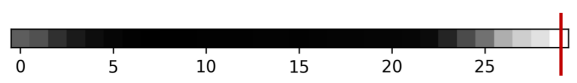

(a) Standard Agents

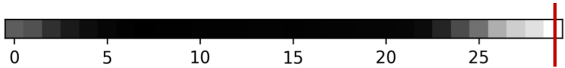

(c) Lazy Speaker + Standard Listener

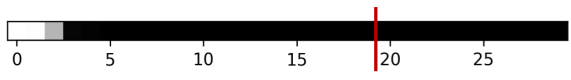

(b) Standard Speaker + Impatient Listener

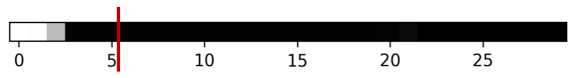

(d) LazImpa

Figure 4: Fraction of informative symbols at each position $\mathrm{k}$ averaged across all emergent messages of successful runs $\left(\left(\Lambda_{k, .}\right)_{0 \leq k \leq 29}\right)$. Each box represents the proportion of informative symbols at a given position $\Lambda_{k, .}$ mapped to a color according to a gray gradient (black $=0$; white $=1$ ). The red vertical lines mark the mean message length $L_{\text {type }}$ across successful runs.

spectrum for LazImpa. Indeed, Impatient Listener relies on $\rho_{\text {inf }}=60 \%$ of the symbols. This corresponds to a big increase compared to $\rho_{\text {inf }}=19 \%$ when using Standard Agents. Yet, we are still far from the $100 \%$ observed in Optimal Coding. That is, even with the introduction of a length cost (with Lazy Speaker), we still encounter non-informative symbols. Finally, these informative symbols are localized in the first positions, opposite to what we observed with Standard Agents. We will show in Section 4.3 how this immediate presence of information is crucial for the length reduction of the messages.

In sum, if we consider only informativeleffective positions, Standard Agents use efficient and ZLA-like (effective) communicative protocol. However, they make it maximally long adding non-informative symbols at the beginning of each message. Introducing LazImpa reverses the length distribution. Indeed, we observe with LazImpa the emergence of efficient and ZLA-obeying languages, with significantly larger $\rho_{i n f}$.

\subsection{LazImpa vs. reference distributions}

We demonstrated above how LazImpa leads to codes with length significantly shorter than the one obtained with Standard Agents.

We compare it here with stricter references, namely natural languages and Optimal Coding. We show that LazImpa results in languages as efficient as natural languages both in terms of length statistics and symbols distribution. However, agents do not manage to reach optimality.

Comparison with natural languages. We see in Figure 5a that the message lengths in the emergent communication are analogous to the words lengths in natural languages: close average $L_{\text {token }}$ and $L_{\text {type }}$ (see Table 1).

We further compare their unigram distributions. Chaabouni et al. (2019) showed that Standard Agents develop repetitive messages with a skewed unigram distribution. Our results, in Figure 5b, show that, on top of a ZLA-like code, LazImpa enables the emergence of natural-language-like unigram distribution, without any particular repetitive pattern. Intriguingly, this similarity with natural languages is an unexpected property as a uniform distribution of unigrams would lead to a more efficient protocol.

Comparison with Optimal Coding. If LazImpa leads to significantly more efficient languages compared to Standard Agents, these emergent languages are still not as efficient as Optimal Coding (see Figure 3). One obvious source of sub-optimality is the addition of uninformative symbols at the end of the messages (i.e. the difference between $L_{e f f}=2.67$ and $L_{\text {type }}-1=4.49$ ). Interestingly, when analyzing the intermediate predictions of Impatient Listener, we see that this model is actually able to guess the right input only reading approximately the $L_{\text {eff }}$ first positions (see Appendix A.4.1 for details). However, we still can note that the informative length $L_{e f f}$ is slightly sub-optimal ( $L_{e f f}=2.67$ for LazImpa, $L_{\text {eff }}=1.96$ for Optimal Coding). This difference 

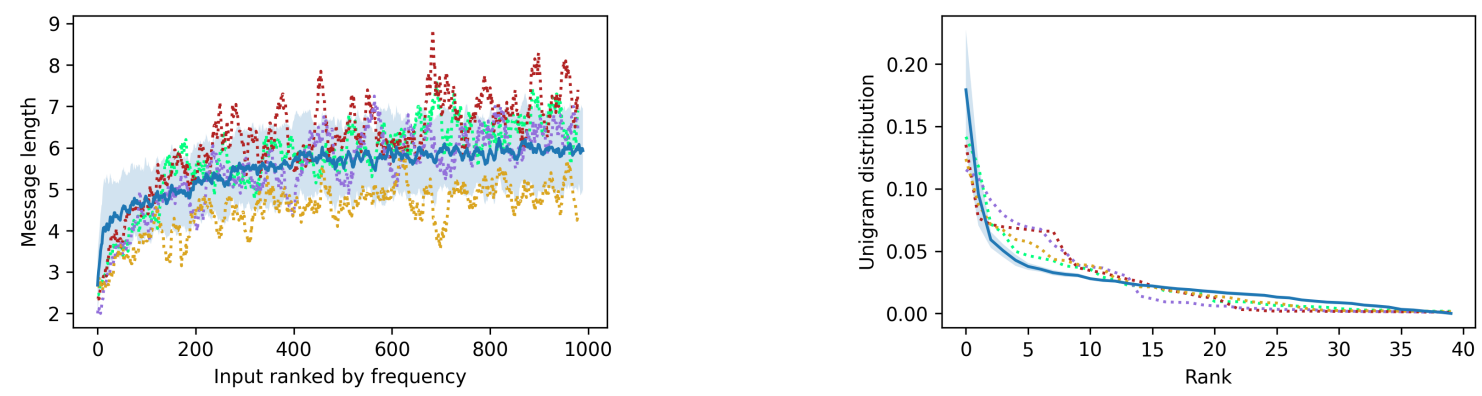

English $\quad \cdots .$. Spanish

Russian

Arabic Lazlmpa

(a) Message length of natural languages and LazImpa (averaged across successful runs) as a function of input frequency rank. For readability, the curves have been smoothed using a sliding average of 20 consecutive lengths, see the real curves in Appendix A.4.3. The light blue interval shows 1 standard deviation for LazImpa's distribution.

(b) Unigrams distribution of natural languages and LazImpa (averaged across successful messages) ranked by unigram frequency. The light blue interval shows 1 standard deviation for LazImpa's unigrams distribution.

Figure 5: Comparison of LazImpa' statistics and natural languages.

can be explained by the non-uniform use of unigrams Specifically, we show in Appendix A.4.1 that effective lengths of LazImpa messages approximate Optimal Coding when the latter uses the same skewed unigram distribution.

\subsection{Ablation study}

We have just seen that our new communication system LazImpa allows agents to develop an efficient and ZLAobeying language whose statistical properties are close to those of natural languages. In this section, we analyze the effects of the modeling choices we have made.

We first look at the effect of Laziness. To do so, we compare LazImpa to the system "Standard Speaker + Impatient Listener" (i.e. removing the length regularization). Figure 6a shows the joint evolution of the mean length of messages $\left(L_{\text {type }}\right)$ and game accuracy. We observe that our non-regularized system, similarly to LazImpa, initially explores long messages while being more successful (exploration step). Surprisingly, even in the absence of Laziness, the exploration step does not continue to maximally long messages, as it is the case for Standard Agents, but breaks at length $\approx 20$. However, contrary to LazImpa, "Standard Speaker + Impatient Listener" does not present a reduction step (a reduction of mean length for a fixed good accuracy). Thus, as expected, the introduction of Laziness in LazImpa is responsible for the reduction step, and hence for a shorter and more efficient communication protocol. However, we note in Figure 6b, that Impatience alone is sufficient for the emergence of ZLA. Moreover, when looking at the information spectrum, comparing "Standard Speaker + Impatient Listener" (Figure 4b) to LazImpa (Figure 4d), we observe how alike both systems allocate information and differ only by their mean length.

Second, we investigate the role of Impatience. We see in Figure 6a that the system "Lazy Speaker + Standard Listener" admits a visually different dynamic compared to LazImpa. In particular, the exploration step leads to significantly longer messages, close to max_len. Interestingly, if we demonstrated above the necessity of Laziness for the reduction step, alone, it does not induce it: no reduction step in the "Lazy Speaker + Standard Listener" system is observed. This is due to the necessity of long messages when experimenting with Standard Listener. Specifically, as informative symbols are present only at the last positions (see Figure 4c), introducing a length regularization provokes a drop in accuracy, which in turn cancels the regularization. In other words, the length regularization scheduling stops at the exploration step, which makes the system almost equivalent to Standard Agents (this could be also seen experimentally in Figures $6 a$ and $6 b$ ).

Taken together, our analysis emphasizes the importance of both Impatience and Laziness for the emergence of efficient communication.

\section{Conclusion}

We demonstrated that a standard communication system, where standard Speaker and Listener LSTMs are trained to solve a simple reconstruction game, leads to long messages, close to the maximal threshold. Surprisingly, if these messages are long, LSTM agents rely only on a small number of informative message symbols, located at the end. We then introduce LazImpa, a constrained system that consists of Lazy Speaker and Impatient Listener. On the one hand, Lazy Speaker is obtained by introducing a cost on messages length once the communication is successful. We found that early exploration of potentially long messages is crucial for successful convergence (similar to the exploration in RL settings). On the other hand, Impatient Listener aims to succeed at the game as soon as possible, by predicting Speaker's input at each message's symbol.

We show that both constraints are necessary for the emergence of a ZLA-like protocol, as efficient as natural languages. Specifically, Lazy Speaker alone would 

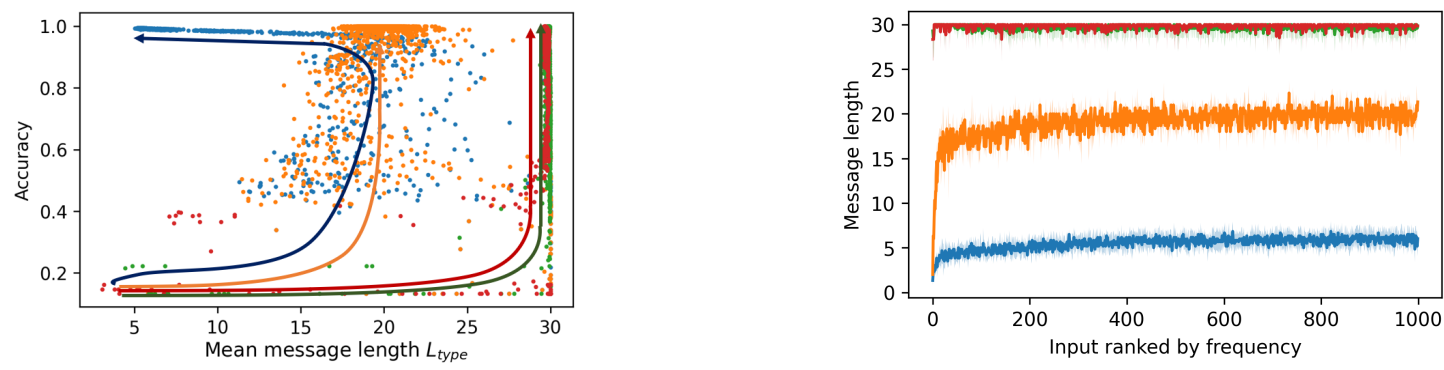

— LazImpa _ Standard Speaker + Impatient Listener

(a) Joint evolution of the accuracy and mean length for the different models. Each point shows the couple ( $L_{\text {type }}$,accuracy) of one training episode. Arrows represent the average joint evolution of the two variables.

(b) Average message length as a function of input frequency rank for the different systems. Light color intervals show 1 standard deviation.

Figure 6: Comparison of different communication systems.

fail to shorten the messages. We connect this to the importance of the Impatience mechanism to locate useful information at the beginning of the messages. If the function of this mechanism is subject to a standing debate (e.g., Jackendoff, 2007; Anderson and Chemero, 2013), many prior works had pointed to its necessity to human language understanding (e.g., Friston, 2010; Clark, 2013). We augment this line of works and suggest that impatience could be at play in the emergence of ZLA-obeying languages. However, if impatience leads to ZLA, it is not sufficient for human-level efficiency. In other words, efficiency needs constraints both on Speaker and Listener sides.

Our work highlights the importance of introducing the right pressures in the communication system. Indeed, to construct automated agents that would eventually interact with humans, we need to introduce taskagnostic constraints, allowing the emergence of more human-like communication. Moreover, while being general, LazImpa provides a more stable optimization compared to the unconstrained system. Finally, this study opens several lines of research. One would be to investigate further the gap from optimality. Indeed, while LazImpa emergent languages show human-level efficiency, they do not reach optimal coding. Specifically, emergent languages still have non-informative symbols at the end of the messages. If these additional non-useful symbols drift the protocol from optimality, we encounter similar trend in human (Marslen-Wilson, 1987) and animal communication (McLachlan and Magrath, 2020). We leave the understanding of the role of these non-informative symbols and how we can reach optimal coding for future works. A second line of research would be to apply this system to other games or NLP problems and study how it affects other properties of the language such as regularity or compositionality.

\section{Acknowledgments}

We would like to thank Emmanuel Chemla, Marco Baroni, Eugene Kharitonov, and the anonymous reviewers for helpful comments and suggestions.
This work was funded in part by the European Research Council (ERC-2011-AdG-295810 BOOTPHON), the Agence Nationale pour la Recherche (ANR-17-EURE-0017 Frontcog, ANR-10-IDEX-000102 PSL*, ANR-19-P3IA-0001 PRAIRIE 3IA Institute) and grants from CIFAR (Learning in Machines and Brains), Facebook AI Research (Research Grant), Google (Faculty Research Award), Microsoft Research (Azure Credits and Grant), and Amazon Web Service (AWS Research Credits).

\section{References}

Gerry TM Altmann and Jelena Mirković. 2009. Incrementality and prediction in human sentence processing. Cognitive science, 33(4):583-609.

Michael L Anderson and Tony Chemero. 2013. The problem with brain guts: Conflation of different senses of" prediction" threatens metaphysical disaster. Behavioral and Brain Sciences, 36(3):204.

Diane Bouchacourt and Marco Baroni. 2018. How agents see things: On visual representations in an emergent language game. pages 981-985.

Rahma Chaabouni, Eugene Kharitonov, Diane Bouchacourt, Emmanuel Dupoux, and Marco Baroni. 2020. Compositionality and generalization in emergent languages. arXiv preprint arXiv:2004.09124.

Rahma Chaabouni, Eugene Kharitonov, Emmanuel Dupoux, and Marco Baroni. 2019. Anti-efficient encoding in emergent communication.

Andy Clark. 2013. Whatever next? predictive brains, situated agents, and the future of cognitive science. Behavioral and brain sciences, 36(3):181-204.

Thomas Cover and Joy Thomas. 2006. Elements of Information Theory, 2nd ed. Wiley, Hoboken, NJ.

Kara D Federmeier. 2007. Thinking ahead: The role and roots of prediction in language comprehension. Psychophysiology, 44(4):491-505. 
Ramon Ferrer i Cancho, Antoni Hernández-Fernández, David Lusseau, Govindasamy Agoramoorthy, Minna Hsu, and Stuart Semple. 2013. Compression as a universal principle of animal behavior. Cognitive Science, 37(8):1565-1578.

Karl Friston. 2010. The free-energy principle: a unified brain theory? Nature reviews neuroscience, 11(2):127-138.

Edward Gibson, Richard Futrell, Steven P Piantadosi, Isabelle Dautriche, Kyle Mahowald, Leon Bergen, and Roger Levy. 2019. How efficiency shapes human language. Trends in cognitive sciences, 23(5):389407.

Serhii Havrylov and Ivan Titov. 2017. Emergence of language with multi-agent games: Learning to communicate with sequences of symbols. pages 21492159.

Sepp Hochreiter and Jürgen Schmidhuber. 1997. Long short-term memory. Neural computation, 9(8):17351780.

Ray Jackendoff. 2007. A parallel architecture perspective on language processing. Brain research, 1146:222 .

Jasmeen Kanwal, Kenny Smith, Jennifer Culbertson, and Simon Kirby. 2017. Zipf's law of abbreviation and the principle of least effort: Language users optimise a miniature lexicon for efficient communication. Cognition, 165:45-52. Copyright (C) 2017 Elsevier B.V. All rights reserved.

Eugene Kharitonov, Rahma Chaabouni, Diane Bouchacourt, and Marco Baroni. 2019. EGG: a toolkit for research on emergence of language in games. In Proceedings of EMNLP (System Demonstrations).

Diederik P. Kingma and Jimmy Ba. 2014. Adam: A method for stochastic optimization.

Simon Kirby. 2001. Spontaneous evolution of linguistic structure-an iterated learning model of the emergence of regularity and irregularity. IEEE Transactions on Evolutionary Computation, 5(2):102-110.

Satwik Kottur, José Moura, Stefan Lee, and Dhruv Batra. 2017. Natural language does not emerge 'naturally' in multi-agent dialog.

Angeliki Lazaridou, Karl Moritz Hermann, Karl Tuyls, and Stephen Clark. 2018. Emergence of linguistic communication from referential games with symbolic and pixel input.

Angeliki Lazaridou, Alexander Peysakhovich, and Marco Baroni. 2017. Multi-agent cooperation and the emergence of (natural) language. Published online: https://openreview. net/group?id= ICLR.CC/2017/conference.

David Lewis. 1969. Convention. Harvard University Press, Cambridge, MA.
William D Marslen-Wilson. 1987. Functional parallelism in spoken word-recognition. Cognition, 25(12):71-102.

Jessica R McLachlan and Robert D Magrath. 2020. Speedy revelations: how alarm calls can convey rapid, reliable information about urgent danger. Proceedings of the Royal Society B, 287(1921):20192772.

Steven T Piantadosi, Harry Tily, and Edward Gibson. 2011. Word lengths are optimized for efficient communication. Proceedings of the National Academy of Sciences, 108(9):3526-3529.

John Schulman, Nicolas Heess, Theophane Weber, and Pieter Abbeel. 2015. Gradient estimation using stochastic computation graphs. In Proceedings of the 28th International Conference on Neural Information Processing Systems - Volume 2, page 3528-3536, Cambridge, MA, USA. MIT Press.

Bengt Sigurd, Mats Eeg-Olofsson, and Joost Van Weijer. 2004. Word length, sentence length and frequencyzipf revisited. Studia Linguistica, 58(1):37-52.

Udo Strauss, Peter Grzybek, and Gabriel Altmann. 2007. Word length and word frequency. In Contributions to the science of text and language, pages 277-294. Springer.

William J Teahan, Yingying Wen, Rodger McNab, and Ian H Witten. 2000. A compression-based algorithm for chinese word segmentation. Computational Linguistics, 26(3):375-393.

Ronald Williams and Jing Peng. 1991. Function optimization using connectionist reinforcement learning algorithms. Connection Science, 3:241-.

Ronald J. Williams. 1992. Simple statistical gradientfollowing algorithms for connectionist reinforcement learning. Machine Learning, 8(3):229-256.

George Zipf. 1949. Human Behavior and the Principle of Least Effort. Addison-Wesley, Boston, MA.

George Kingsley Zipf. 2013. The psycho-biology of language: An introduction to dynamic philology. Routledge. 